\title{
Guest Removal and External Pressure Variation Induce Spin Crossover in Halogen-Functionalized 2-D Hofmann Frameworks
}

Ashley T. Brennan, Katrina A. Zenere, Helen E. A. Brand, Jason R. Price, Mohan M. Bhadbhade, Gemma F. Turner, Stephen A. Moggach, Francisco-Javier Valverde-Muñoz, Jose A. Real, Jack K. Clegg, Cameron J. Kepert and Suzanne M. Neville*

Ashley T. Brennan - The School of Chemistry, UNSW Sydney, Sydney 2052, New South Wales, Australia.

Katrina A. Zenere - The School of Chemistry, The University of Sydney, Sydney 2006, New South Wales, Australia. Helen E. A. Brand - Australian Synchrotron, ANSTO Clayton 3800, Victoria, Australia.

Jason R. Price - The School of Chemistry, UNSW Sydney, Sydney 2052, New South Wales, Australia; Australian Synchrotron, ANSTO Clayton 3800, Victoria, Australia.

Mohan M. Bhadbhade - Mark Wainwright Analytical Centre, UNSW Sydney, Sydney 2052, New South Wales, Australia. Gemma F. Turner - School of Molecular Sciences/Centre for Microscopy, Characterisation and Analysis, The University of Western Australia, Perth 6009, Western Australia, Australia.

Stephen A. Moggach - School of Molecular Sciences/Centre for Microscopy, Characterisation and Analysis, The University of Western Australia, Perth 6009, Western Australia, Australia.

Francisco J. Valverde-Muñoz - Institut the Ciencia Molecular, Department de Quimica Inorganica, Universitat de Valéncia, Patterna 46980, Valéncia, Spain.

Jose A. Real - Institut the Ciencia Molecular, Department de Quimica Inorganica, Universitat de Valéncia, Patterna 46980, Valéncia, Spain.

Jack K. Clegg - School of Chemistry and Molecular Biosciences, The University of Queensland, St Lucia 4072, Queensland, Australia.

Cameron J. Kepert - The School of Chemistry, The University of Sydney, Sydney 2006, New South Wales, Australia. Suzanne M. Neville - The School of Chemistry, UNSW Sydney, Sydney 2052, New South Wales, Australia.

s.neville@unsw.edu.au.

\section{Contents}

S1. Thermogravimetric analysis (TGA)

S2. Variable temperature single crystal diffraction

S3. Temperature dependent magnetic susceptibility

S4. Variable temperature synchrotron powder diffraction

S5. Variable pressure magnetic susceptibility

S6. Variable pressure single crystal analysis 


\section{S1. Thermogravimetric analysis (TGA)}

TGA was carried out for both $\mathbf{1}^{\mathrm{Cl}} \cdot \mathbf{2} \mathrm{H}_{2} \mathrm{O}$ and $\mathbf{2}^{\mathrm{Br}} \cdot \mathbf{2} \mathrm{H}_{2} \mathrm{O}$ to assess the solvent removal and decomposition process (Fig. S1). The complexes show a mass decrease in the temperature range $100^{\circ} \mathrm{C}$ to $200^{\circ} \mathrm{C}$ of $5.06 \%$ and $4.74 \%$, respectively. This corresponds with the loss of all the solvent water from the pores of the framework and compares well with the theoretical values of $4.95 \%$ and $4.41 \%$, respectively. For both analogues, decomposition of the framework components occurs beyond $200^{\circ} \mathrm{C}$.

(a)

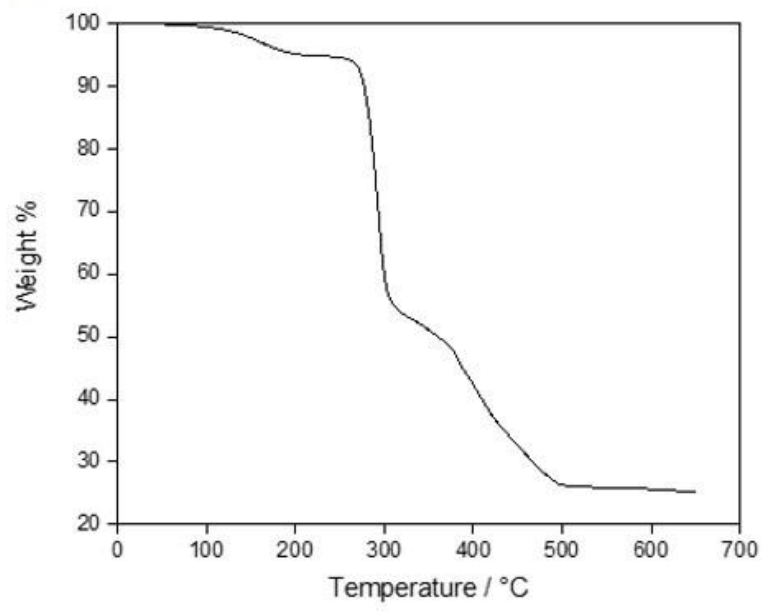

(b)

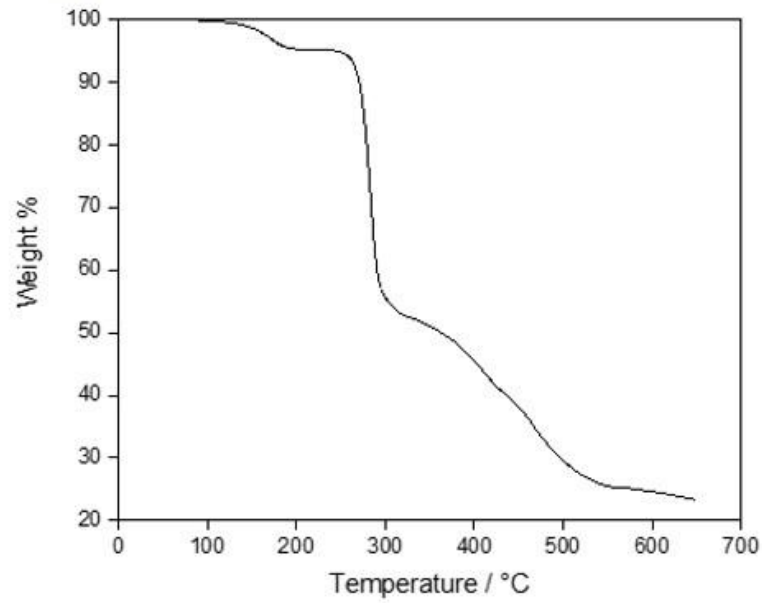

Figure S1. TGA for $\mathbf{1}^{\mathrm{Cl}} \cdot 2 \mathrm{H}_{2} \mathrm{O}$ and $\mathbf{2}^{\mathrm{Br}} \cdot \mathbf{2} \mathrm{H}_{2} \mathrm{O}$. 


\section{S2: Variable temperature single crystal diffraction}

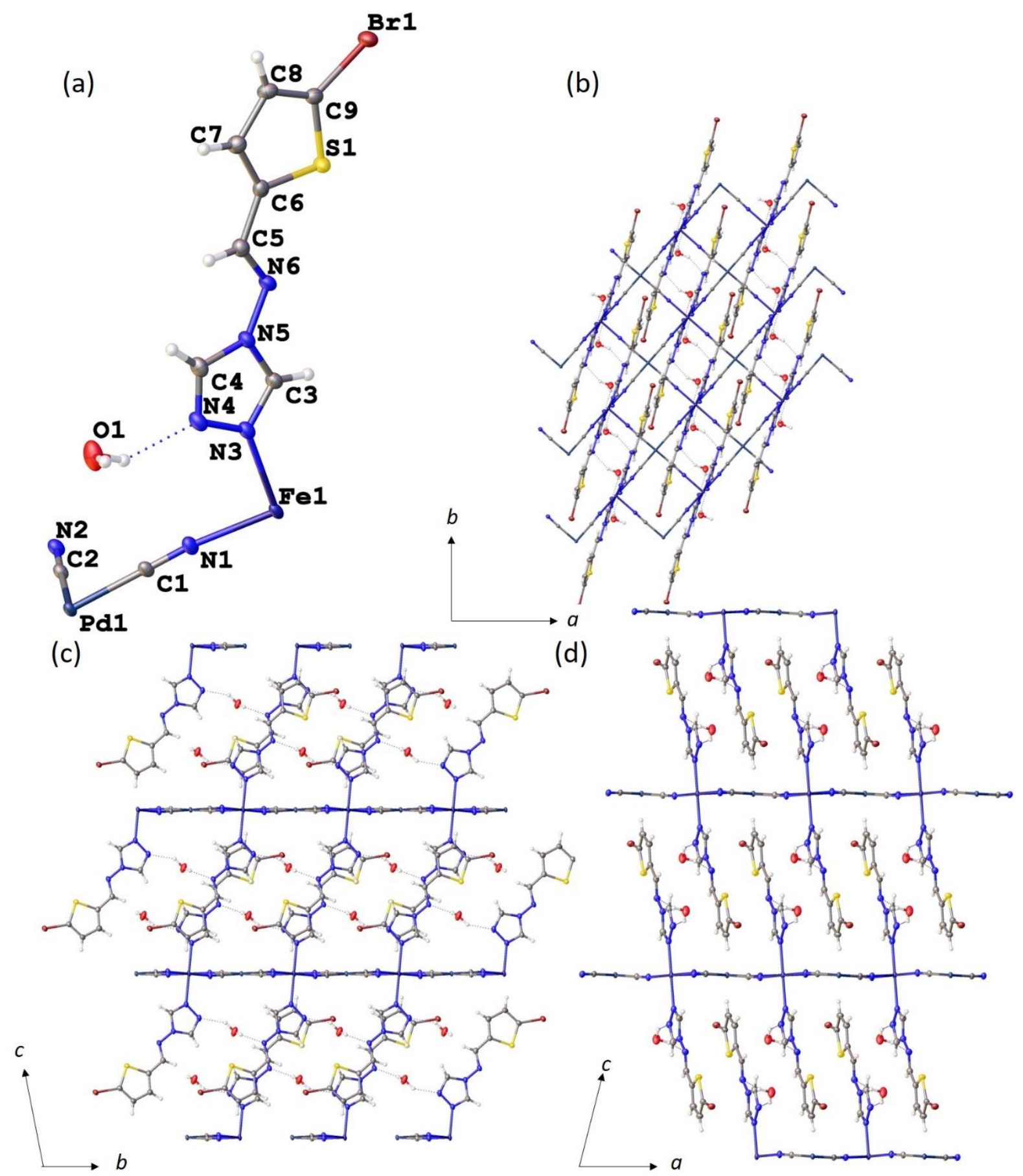

Figure S2: (a) The asymmetric unit of $\mathbf{2}^{\mathrm{Br}} \cdot \mathbf{2} \mathrm{H}_{2} \mathbf{O}$ (100 K), showing the thioBr ligand which binds to Fe1 via $\mathrm{N} 3$, the $\left[\mathrm{Pd}(\mathrm{CN})_{4}\right]$ anion bound to $\mathrm{Fe} 1$ via the $\mathrm{N}$ atoms and the hydrogen bound water molecule; Views of the packing along the (001), (100) and (010) of the $\mathbf{2}^{\mathrm{Br}} \cdot \mathbf{2} \mathbf{H}_{2} \mathbf{O}$ crystal structure (100 K) showing the (b) Hofmann grids, (c) the layer stacking, overlapped thioBr ligands and water molecules in the interlayer spacing and (d) The interdigitated packing of thioBr ligands from adjacent layers. 
(a)

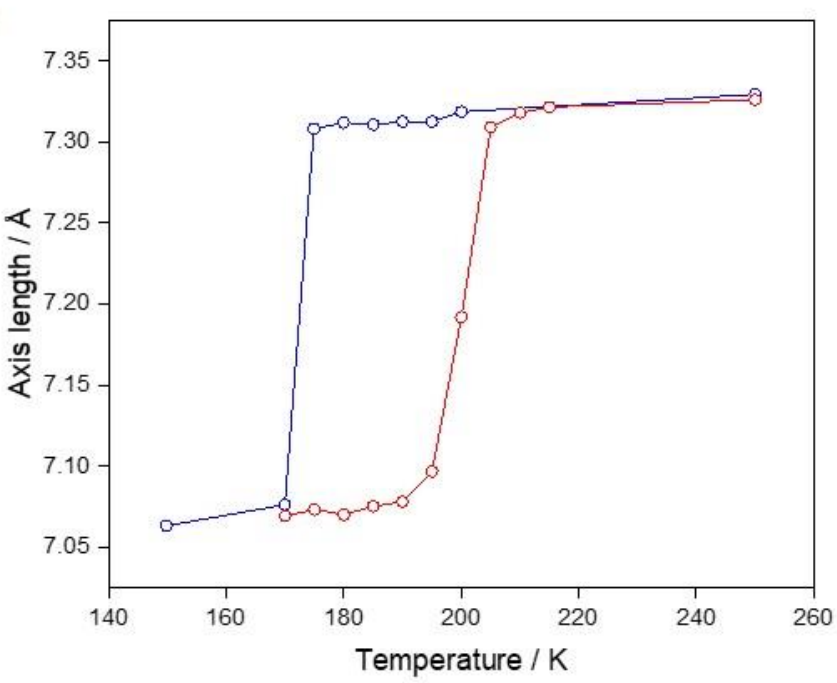

(b)

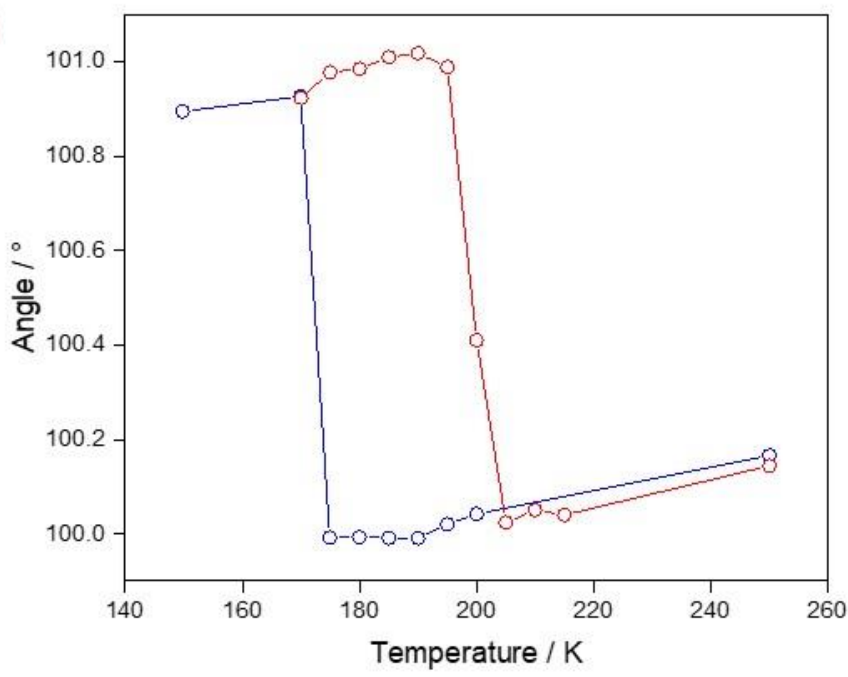

(c)

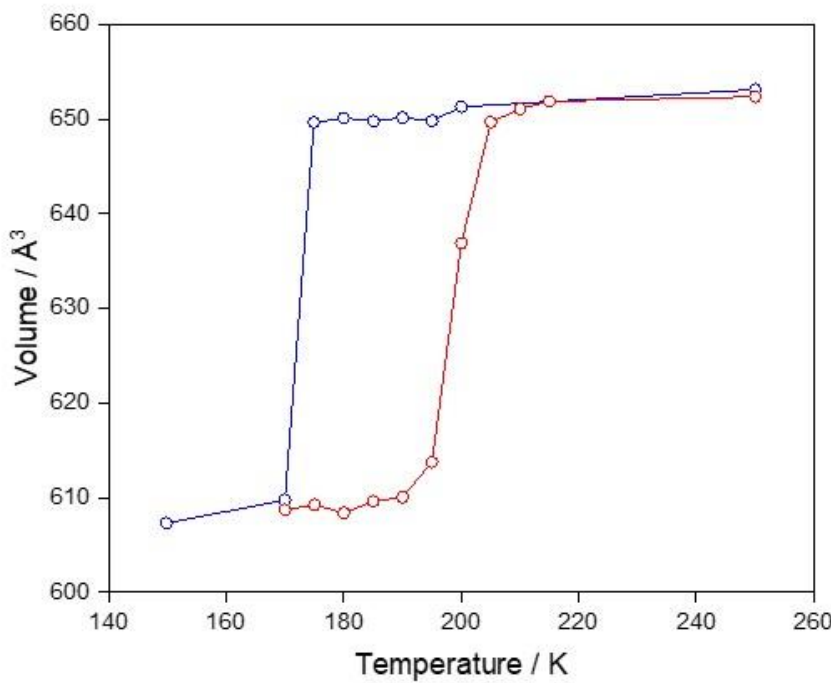

Figure S3. Unit cell parameters from variable temperature single crystal X-ray diffraction analysis of $\mathbf{1}^{\mathrm{Cl}}$ showing (a) $a$-axis, (b) $\beta$ angle and (c) unit cell volume. Blue $=$ cooling, red $=$ heating. 
(a)

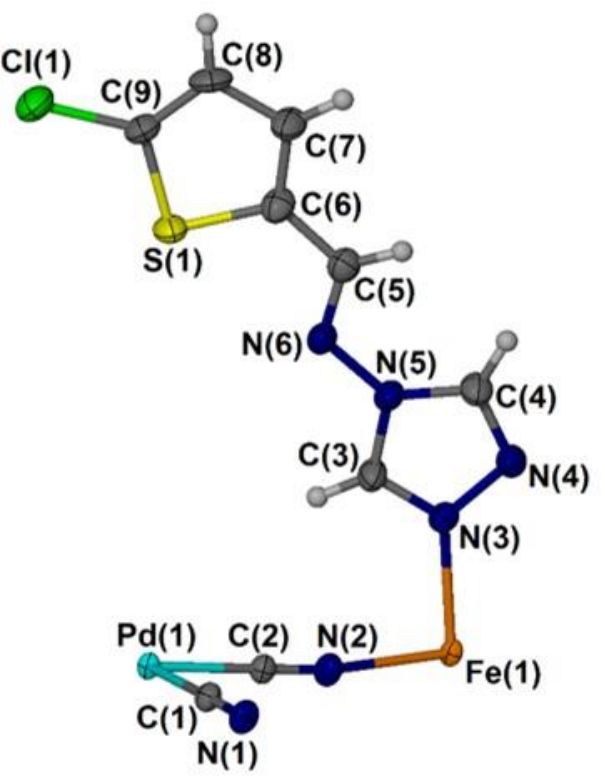

(c)

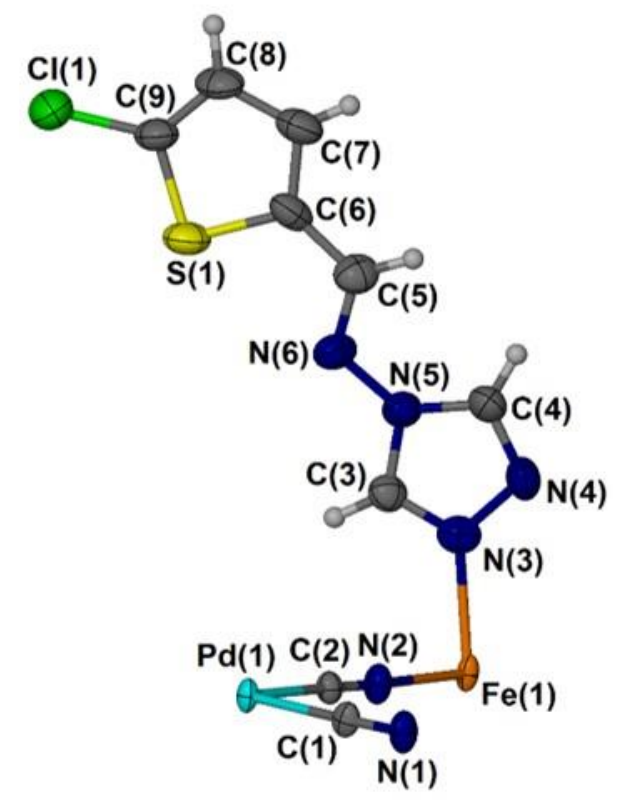

(b)

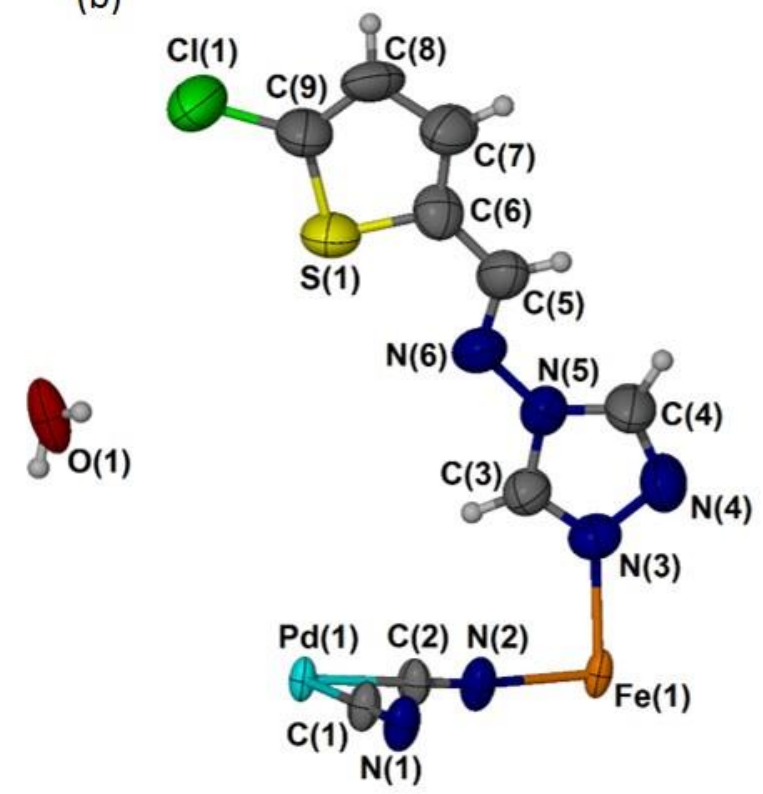

(d)

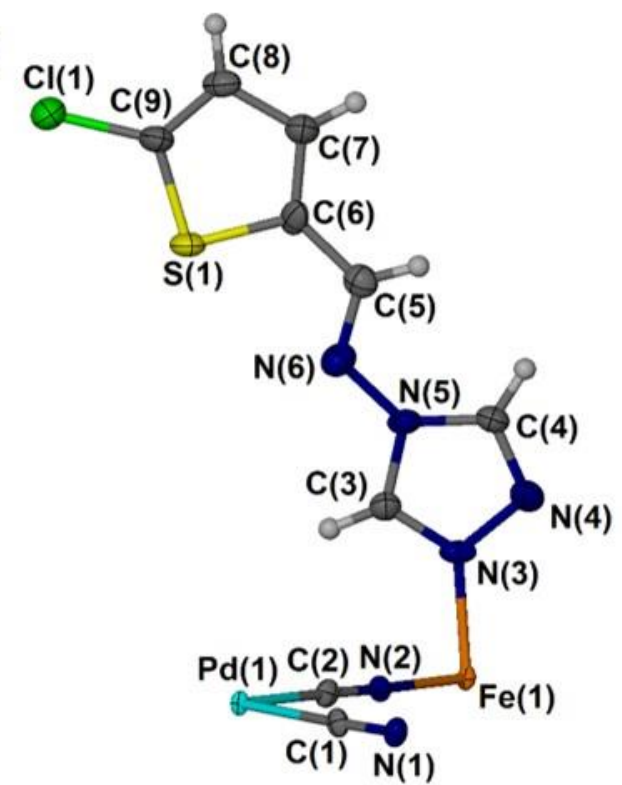

Figure S4. The ASU for $\mathbf{1}^{\mathrm{Cl}} \cdot \mathbf{2} \mathrm{H}_{2} \mathbf{O}$ at (a) $250 \mathrm{~K}$ and $\mathbf{1}^{\mathrm{cl}}$ at (b) $450 \mathrm{~K}$, (c) $250 \mathrm{~K}$ (HS state) and (d) $150 \mathrm{~K}$ (LS state). 
Table S1. Crystal data for $\mathbf{1}^{\mathrm{Cl}} \cdot \mathbf{2} \mathrm{H}_{2} \mathrm{O}$ and $\mathbf{2}^{\mathrm{Br}} \cdot \mathbf{2} \mathrm{H}_{2} \mathrm{O}$.

\begin{tabular}{|c|c|c|}
\hline Parameter & $1^{\mathrm{Cl}} \cdot 2 \mathrm{H}_{2} \mathrm{O}$ & $2^{\mathrm{Br}} \cdot \mathbf{2} \mathrm{H}_{2} \mathrm{O}$ \\
\hline $\mathrm{Fe}^{\mathrm{II}}$ spin state & HS & HS \\
\hline Empirical formula & $\mathrm{C}_{18} \mathrm{H}_{12} \mathrm{Cl}_{2} \mathrm{FeN}_{12} \mathrm{PdS}_{2} \mathrm{O}$ & $\mathrm{C}_{18} \mathrm{H}_{12} \mathrm{Br}_{2} \mathrm{FeN}_{12} \mathrm{PdS}_{2} \mathrm{O}$ \\
\hline Formula weight/ $\mathrm{g} \mathrm{mol}^{-1}$ & 727.68 & 816.60 \\
\hline $\mathrm{T} / \mathrm{K}$ & 100 & 100 \\
\hline Crystal system & Triclinic & Triclinic \\
\hline Space group & $P-1$ & $P-1$ \\
\hline$a / \AA$ & $7.2890(5)$ & $7.2960(15)$ \\
\hline$b / \AA ̊$ & $7.5949(5)$ & $7.5950(15)$ \\
\hline$c / \AA$ & $12.2858(12)$ & $12.529(3)$ \\
\hline$\alpha /^{\circ}$ & $105.893(7)$ & 107.05(3) \\
\hline$\beta 1^{\circ}$ & 97.773(7) & $97.82(3)$ \\
\hline$\gamma /{ }^{\circ}$ & $90.034(5)$ & $90.08(3)$ \\
\hline Volume $/ \AA^{3}$ & $647.62(9)$ & $657.0(3)$ \\
\hline $\mathrm{Z}$ & 1 & 1 \\
\hline$\rho_{\text {calc }} \mathrm{g} / \mathrm{cm}^{3}$ & 1.835 & 2.064 \\
\hline$\mu / \mathrm{mm}^{-1}$ & 1.665 & 4.480 \\
\hline Data/restraints/parameters & $6622 / 3 / 182$ & 3947 / 0 / 181 \\
\hline Goodness-of-fit on $\mathrm{F}^{2}$ & 1.111 & 1.056 \\
\hline Final $\mathrm{R}$ indexes $[\mathrm{I}>=2 \sigma(\mathrm{I})]$ & $\mathrm{R}_{1}=0.0795, \mathrm{wR}_{2}=0.2026$ & $\mathrm{R}_{1}=0.0341, \mathrm{wR}_{2}=0.0904$ \\
\hline Final $\mathrm{R}$ indexes [all data] & $\mathrm{R}_{1}=0.1124, \mathrm{wR}_{2}=0.2323$ & $\mathrm{R}_{1}=0.0351, \mathrm{wR}_{2}=0.0930$ \\
\hline
\end{tabular}

Table S2. Summary of selected structural parameters for $\mathbf{1}^{\mathrm{Cl}} \cdot \mathbf{2} \mathrm{H}_{2} \mathbf{O}$ and $\mathbf{2}^{\mathrm{Br}} \cdot \mathbf{2} \mathrm{H}_{2} \mathbf{O}$.

\begin{tabular}{lcc}
\hline Selected parameters & $\mathbf{1}^{\mathrm{Cl} \cdot 2 \mathbf{H}_{2} \mathbf{O}}$ & $\mathbf{2}^{\mathrm{Br} \cdot} \cdot \mathbf{2} \mathbf{H}_{2} \mathbf{O}$ \\
\hline Temperature/K & 100 & 100 \\
$d_{<\mathrm{Fe}-\mathrm{N}>} / \AA$ & $2.164(6)$ & $2.166(2)$ \\
$d_{<\mathrm{Fe}-\mathrm{N}(\mathrm{Py})} / \AA$ & $2.224(6)$ & $2.233(2)$ \\
$d_{<\mathrm{Fe}-\mathrm{N}(\mathrm{CN})>} / \AA$ & $2.135(6)$ & $2.133(2)$ \\
Host-Guest $(\mathrm{N}-\mathrm{O}) / \AA$ & $2.856(7)$ & $2.862(3)$ \\
$\Sigma^{\mathrm{Fe} /} /{ }^{\circ}$ & 25.6 & 24.6 \\
$\mathrm{Fe}-\mathrm{N}-\mathrm{N} /{ }^{\circ}$ & $128.4(5)$ & $129.3(16)$ \\
Average $\mathrm{Fe}-\mathrm{N}-\mathrm{C} /{ }^{\circ}$ & $174.2(6)$ & $175.5(2)$ \\
\hline
\end{tabular}

a Octahedral distortion $\left(\Sigma^{\mathrm{Fe}}\right)$ : sum of the deviation of the 12 cis- $\mathrm{N}-\mathrm{Fe}-\mathrm{N}$ angles from $90^{\circ} 1$ 
Table S3 Single crystal data for structures of $\mathbf{1}^{\mathrm{Cl}} \cdot \mathbf{2} \mathrm{H}_{\mathbf{2}} \mathrm{O}(250 \mathrm{~K})$ and $\mathbf{1}^{\mathrm{Cl}}(450,250$ and $150 \mathrm{~K})$.

\begin{tabular}{|c|c|c|c|c|}
\hline Parameter & $1^{\mathrm{Cl}} \cdot 2 \mathrm{H}_{2} \mathrm{O}$ & $\mathbf{1}^{\mathrm{Cl}}$ & $\mathbf{1}^{\mathrm{Cl}}$ & $\mathbf{1}^{\mathrm{Cl}}$ \\
\hline $\mathrm{Fe}^{\mathrm{II}}$ spin state & $\mathrm{HS}$ & HS & HS & LS \\
\hline Empirical formula & $\begin{array}{c}\mathrm{C}_{18} \mathrm{H}_{14} \mathrm{Cl}_{2} \mathrm{FeN}_{12} \mathrm{PdS} \\
{ }_{2} \mathrm{O}_{2}\end{array}$ & $\mathrm{C}_{18} \mathrm{H}_{10} \mathrm{Cl}_{2} \mathrm{FeN}_{12} \mathrm{PdS}_{2}$ & $\mathrm{C}_{18} \mathrm{H}_{10} \mathrm{Cl}_{2} \mathrm{FeN}_{12} \mathrm{PdS}_{2}$ & $\mathrm{C}_{18} \mathrm{H}_{10} \mathrm{Cl}_{2} \mathrm{FeN}_{12} \mathrm{PdS}_{2}$ \\
\hline Formula weight/ $\mathrm{g} \mathrm{mol}^{-1}$ & 727.68 & 691.61 & 691.61 & 691.61 \\
\hline $\mathrm{T} / \mathrm{K}$ & 250 & 450 & 250 & 150 \\
\hline Crystal system & Triclinic & Triclinic & Triclinic & Triclinic \\
\hline Space group & $P-1$ & $P-1$ & $P-1$ & $P-1$ \\
\hline$a / \AA$ & $7.3399(5)$ & $7.3869(10)$ & $7.3294(5)$ & $7.0633(5)$ \\
\hline$b / \AA$ & $7.5620(5)$ & 7.5293(9) & $7.5438(6)$ & $7.2759(6)$ \\
\hline$c / \AA$ & $12.3718(12)$ & $12.609(2)$ & $12.4033(14)$ & $12.4921(14)$ \\
\hline$\alpha /^{\circ}$ & $106.209(7)$ & $105.130(14)$ & $104.370(8)$ & $105.257(9)$ \\
\hline$\beta /^{\circ}$ & $97.919(7)$ & $100.183(14)$ & $100.166(8)$ & $100.895(8)$ \\
\hline$\gamma /{ }^{\circ}$ & $90.108(5)$ & $90.170(11)$ & $90.143(6)$ & $90.137(6)$ \\
\hline Volume $/ \AA^{3}$ & $653.42(9)$ & $665.43(19)$ & $653.14(10)$ & $607.28(10)$ \\
\hline $\mathrm{Z}$ & 1 & 1 & 1 & 1 \\
\hline$\rho_{\text {calc }} \mathrm{g} / \mathrm{cm}^{3}$ & 1.852 & 1.725 & 1.753 & 1.869 \\
\hline$\mu / \mathrm{mm}^{-1}$ & 1.653 & 1.741 & 1.641 & 1.765 \\
\hline Data/restraints/parameters & 7373 / 3 / 182 & 4342 / 0 / 166 & $4258 / 0 / 169$ & $3910 / 0 / 172$ \\
\hline Goodness-of-fit on $\mathrm{F}^{2}$ & 1.097 & 1.032 & 1.119 & 1.091 \\
\hline Final $\mathrm{R}$ indexes $[\mathrm{I}>=2 \sigma(\mathrm{I})]$ & $\begin{array}{c}\mathrm{R}_{1}=0.0859, \mathrm{wR}_{2}= \\
0.2121\end{array}$ & $\begin{array}{c}\mathrm{R}_{1}=0.0837, \mathrm{wR}_{2}= \\
0.2158\end{array}$ & $\begin{array}{c}\mathrm{R}_{1}=0.0839, \mathrm{wR}_{2}= \\
0.2386\end{array}$ & $\begin{array}{c}\mathrm{R}_{1}=0.0941, \mathrm{wR}_{2}= \\
0.2576\end{array}$ \\
\hline Final $\mathrm{R}$ indexes [all data] & $\begin{array}{c}\mathrm{R}_{1}=0.1394, \mathrm{wR}_{2}= \\
0.2593\end{array}$ & $\begin{array}{c}\mathrm{R}_{1}=0.1476, \mathrm{wR}_{2}= \\
0.2479\end{array}$ & $\begin{array}{c}\mathrm{R}_{1}=0.1203, \mathrm{wR}_{2}= \\
0.2575\end{array}$ & $\begin{array}{c}\mathrm{R}_{1}=0.1206, \mathrm{wR}_{2}= \\
0.2711\end{array}$ \\
\hline
\end{tabular}

Table S4. Selected structural parameters of $\mathbf{1}^{\mathrm{Cl}} \cdot \mathbf{2} \mathbf{H}_{\mathbf{2}} \mathbf{O}(250 \mathrm{~K})$ and $\mathbf{1}^{\mathrm{Cl}}(450,250$ and $150 \mathrm{~K})$.

\begin{tabular}{lcccc}
\hline Selected parameters & $\mathbf{1}^{\mathrm{Cl}} \cdot \mathbf{2} \mathbf{H}_{2} \mathbf{O}$ & $\mathbf{1}^{\mathbf{C l}}$ & $\mathbf{1}^{\mathrm{Cl}}$ & $\mathbf{1}^{\mathbf{C l}}$ \\
\hline $\mathrm{T} / \mathrm{K}$ & 250 & 450 & 250 & 150 \\
$d<\mathrm{Fe}-\mathrm{N}>/ \AA$ & $2.172(6)$ & $2.173(7)$ & $2.161(6)$ & $1.951(8)$ \\
$d<\mathrm{Fe}-\mathrm{N}(\mathrm{Py})>/ \AA$ & $2.224(6)$ & $2.220(8)$ & $2.218(7)$ & $1.975(8)$ \\
$d<\mathrm{Fe}-\mathrm{N}(\mathrm{CN})>/ \AA$ & $2.145(6)$ & $2.150(7)$ & $2.133(6)$ & $1.939(8)$ \\
Host-Guest $(\mathrm{N}-\mathrm{O}) / \AA$ & $2.829(10)$ & - & - & - \\
$\Sigma^{\mathrm{Fe} /}{ }^{\circ}$ & 22.4 & 23.8 & 21.4 & 15.5 \\
$\mathrm{Fe}-\mathrm{N}-\mathrm{N} /{ }^{\circ}$ & $128.6(5)$ & $127.8(7)$ & $128.4(6)$ & $126.2(7)$ \\
Average $\mathrm{Fe}-\mathrm{N}-\mathrm{C} /{ }^{\circ}$ & $175.3(6)$ & $176.1(8)$ & $175.7(7)$ & $176.21(8)$ \\
\hline
\end{tabular}

a Octahedral distortion $\left(\Sigma^{\mathrm{Fe}}\right)$ : sum of the deviation of the 12 cis-N-Fe-N angles from $90^{\circ} 1$ 


\section{S3. Temperature dependent magnetic susceptibility}

(a)

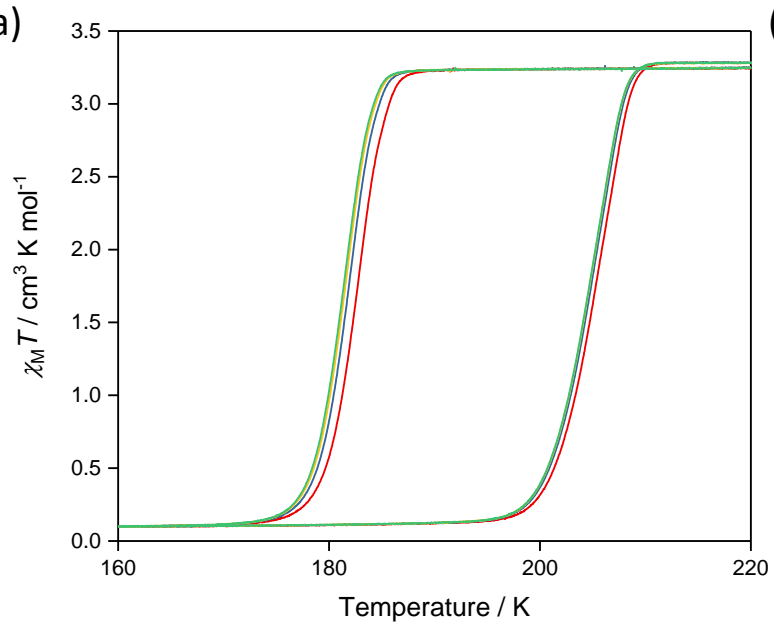

(c)

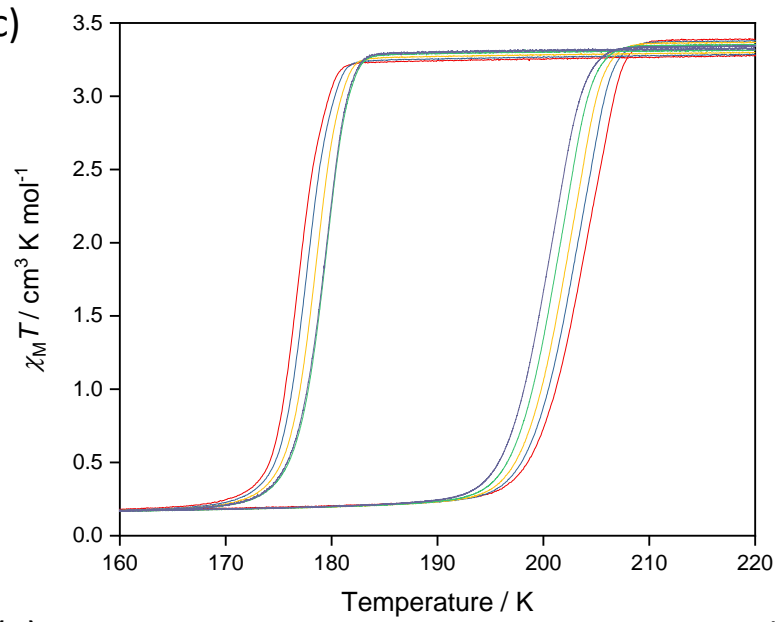

(e)

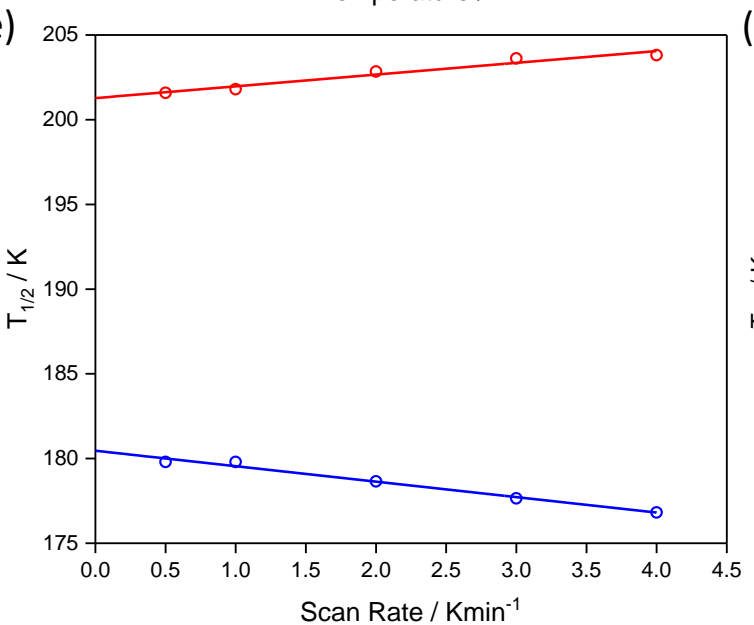

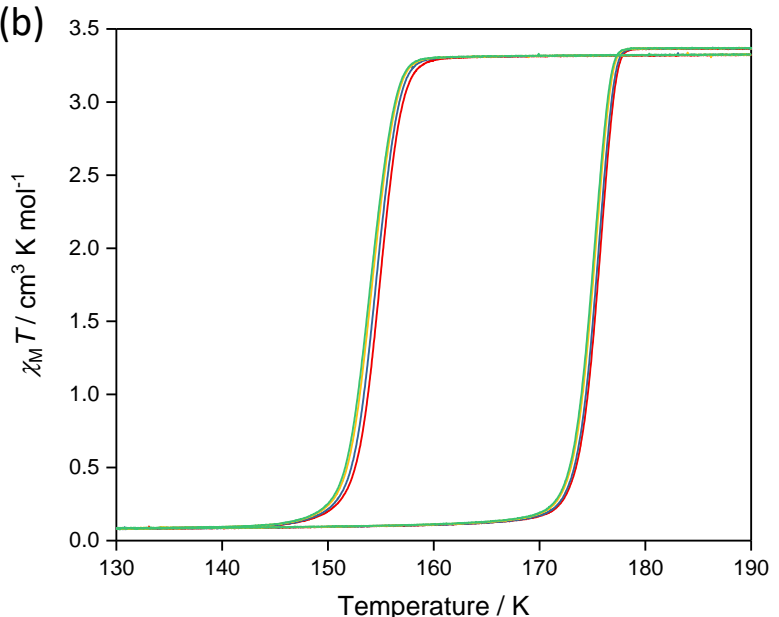

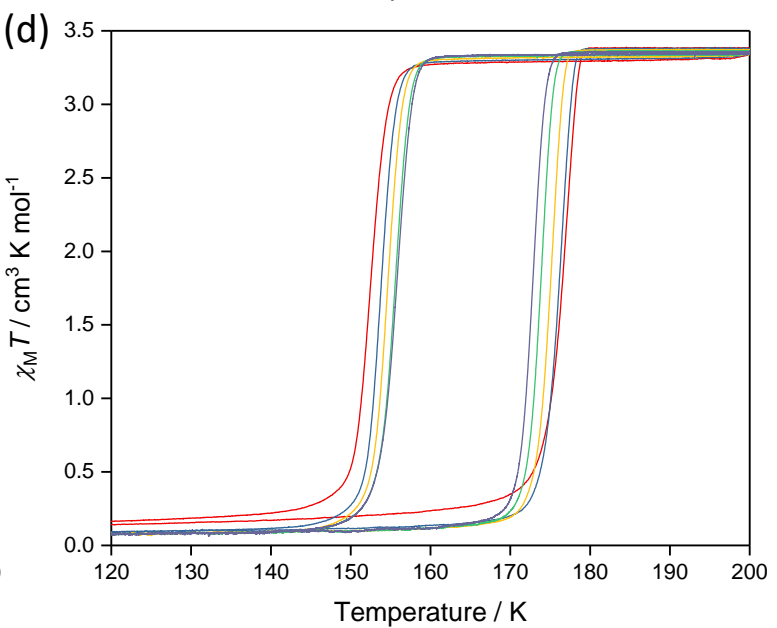

(f)

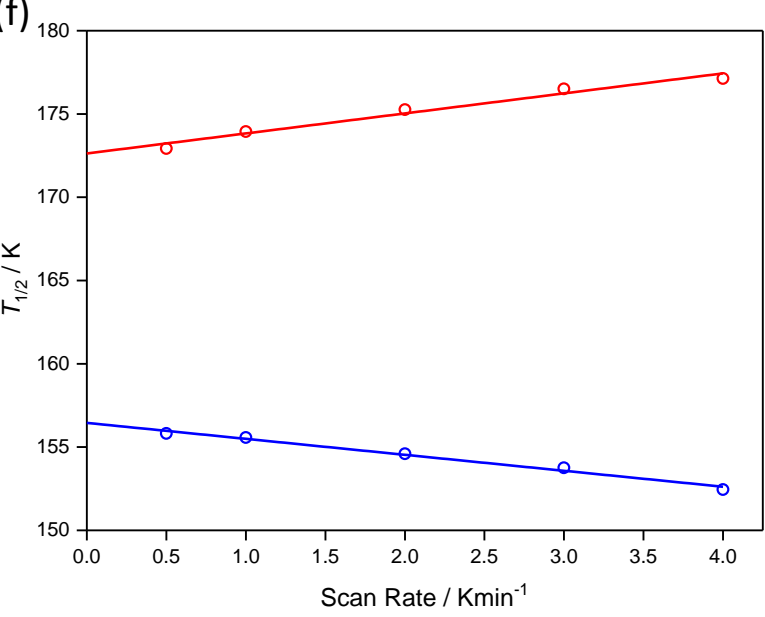

Figure S5. Variable temperature magnetic susceptibility for (a) $\mathbf{1}^{\mathrm{Cl}}$ and (b) $\mathbf{2}^{\mathrm{Br}}$ with cycling. Variable scan rate measurements were also carried out for (c) $\mathbf{1}^{\mathrm{Cl}}$ and (d) $\mathbf{2}^{\mathrm{Br}}$. Red, blue, yellow, green and purple lines show scans at 4, 3, 2, 1 and $0.5 \mathrm{Kmin}^{-1}$ respectively. As scan rate is decreased, the hysteresis band contracts, converging on widths of (e) $20.82 \mathrm{~K}$ for $\mathbf{1}^{\mathrm{Cl}}$ and (f) $16.18 \mathrm{~K}$ for $\mathbf{2}^{\mathrm{Br}}$ when extrapolated to a $0 \mathrm{Kmin}^{-1}$ scan rate. Blue and red symbols show the cooling and heating SCO temperatures respectively, lines show linear fits extrapolated to a 0 $\mathrm{Kmin}^{-1}$ scan rate. 


\section{S4: Variable temperature synchrotron X-ray powder diffraction}

(a)

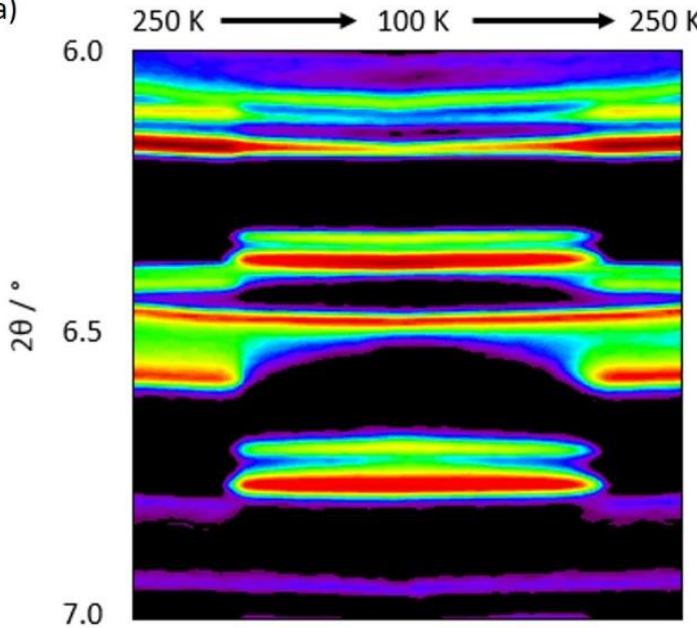

(b)

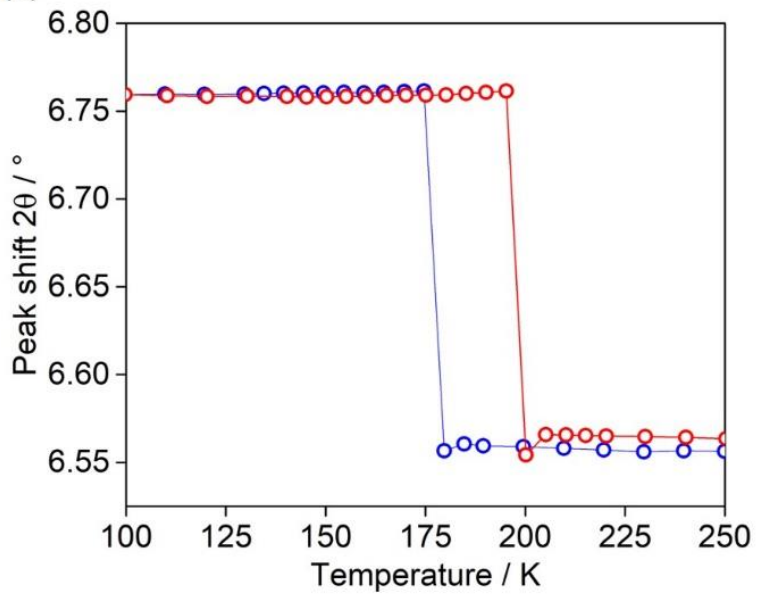

Figure S6. Variable temperature powder X-ray diffraction (250-100-250 K) of $1^{\mathrm{Cl}}$. Note: the sample contains a mixture of $\mathbf{1}^{\mathrm{Cl}} \cdot \mathbf{2} \mathrm{H}_{2} \mathrm{O}$ and $\mathbf{1}^{\mathrm{Cl}}$ due to incomplete desolvation. The peak at $\sim 6.43^{\circ}(1,-1,1$ reflection) remains constant with temperature and is representative of the $\mathbf{1}^{\mathrm{Cl}} \cdot \mathbf{2} \mathrm{H}_{2} \mathrm{O}$ phase, whereas the peak at $\sim 6.58^{\circ}$ (at 250 $\mathrm{K} ; 1,-1,1$ reflection) shifts abruptly over the cooling and heating cycle (HS $\rightarrow$ LS $\rightarrow$ HS state transition). (b) Thermal evolution of the peak positions of the $\mathbf{1}^{\mathrm{Cl}}$ phase extracted from powder X-ray diffraction data peak fit analysis (1,-1,1 reflection); the transition temperatures and SCO transition profile match relatively well with that of the magnetic susceptibly.

(a)

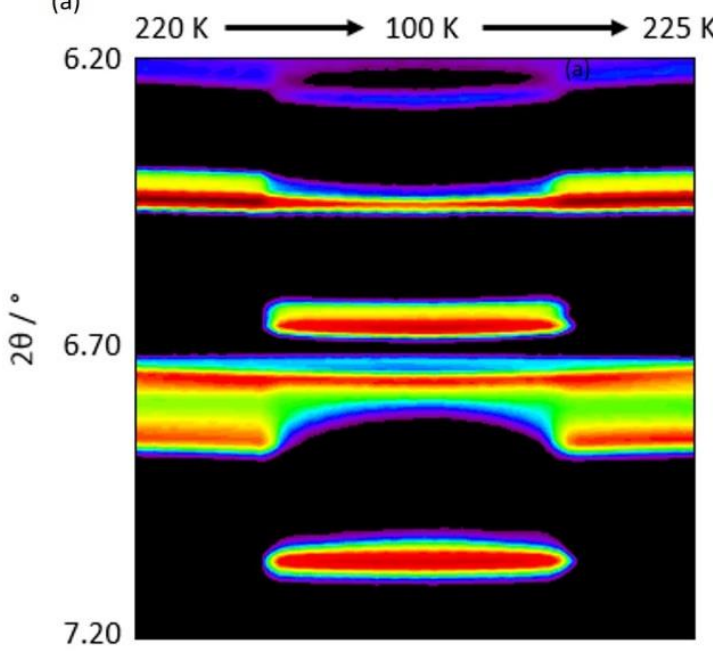

(b)

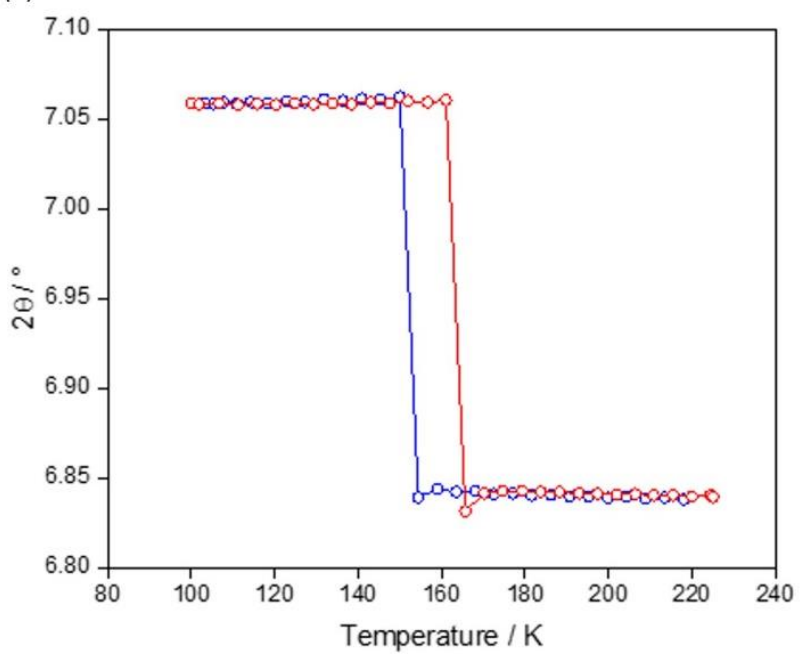

Figure S7. Variable temperature powder X-ray diffraction (220-100-220 K) of $2^{\mathrm{Br}}$ showing the shift in Bragg peaks mimicking the one-step SCO transition of the magnetic data. Note: the sample contains a mixture of $\mathbf{2}^{\mathrm{Br}} \cdot \mathbf{2} \mathbf{H}_{\mathbf{2}} \mathbf{O}$ and $\mathbf{2}^{\mathrm{Br}}$ due to incomplete desolvation. The peak at $\sim 6.72^{\circ}(1,-1,1$ reflection) remains constant with temperature and is representative of the $\mathbf{2}^{\mathrm{Br}} \cdot \mathbf{2} \mathrm{H}_{2} \mathrm{O}$ phase, whereas the peak at $\sim 6.85^{\circ}$ (at $220 \mathrm{~K} ;(1,-1,1$ reflection) shifts abruptly over the cooling and heating cycle (HS $\rightarrow$ LS $\rightarrow$ HS state transition). (b) Thermal evolution of the peak positions of the $2^{\mathrm{Br}}$ phase extracted from powder X-ray diffraction data peak fit analysis $(1,-1,1$ reflection); the transition temperatures and SCO transition profile match relatively well that of the magnetic susceptibly. 


\section{$\underline{\text { S5. Variable pressure magnetic susceptibility }}$}

$T_{1 / 2}^{\text {av }}$ values were estimated as follows: $\left\{\left[\left(\chi_{M} T\right)_{H_{T}-}\left(\chi_{M} T\right)_{L T}\right] / 2\right\}+\left(\chi_{M} T\right)_{L T}$

where $\left(\chi_{M} T\right)_{\mathrm{HT}}$ corresponds to the higher value of $\chi_{M} T$ at high temperature where the compound is essentially $\mathrm{HS} ;\left(\chi_{M} T\right)_{L T}$ is the lowest $\chi_{M} T$ value attained in the low-temperature plateau where no SCO occurs (excluding the interval affected by ZFS). This formula estimates a $\left(\chi_{M} T\right)_{1 / 2}$ value which corresponds to $50 \%$ of the observed SCO. With this value it is possible to obtain the corresponding $T_{1 / 2}$ for the heating and cooling modes when hysteresis is present. Finally, $T_{y / 2}^{a v}$ is obtained as the average value of both temperatures. In the present case the obtained values are as follows:

$p=0.12 \mathrm{GPa} ; T_{1 / 2} \approx 98 \mathrm{~K}, T_{1 / 2}^{\mathrm{av}} \approx 98 \mathrm{~K}$

$p=0.30 \mathrm{GPa} ; T_{1 / 2}($ down $)=161 \mathrm{~K}, T_{1 / 2}$ (up) $=172 \mathrm{~K}, T_{1 / 2}^{\text {av }} \approx 166.5 \mathrm{~K}$

$p=0.48 \mathrm{GPa} ; T_{1 / 2}$ (down) $=232 \mathrm{~K}, T_{1 / 2}$ (up) $=251 \mathrm{~K}, T_{1 / 2}^{\text {av }} \approx 241.5 \mathrm{~K}$

$p=0.62 \mathrm{GPa} ; T_{1 / 2}($ down $)=285 \mathrm{~K}, T_{1 / 2}$ (up) $=295 \mathrm{~K}, T_{1 / 2}^{\text {av }} \approx 290 \mathrm{~K}$ 


\section{$\underline{\text { S6. Variable pressure single crystal X-ray diffraction analysis }}$}
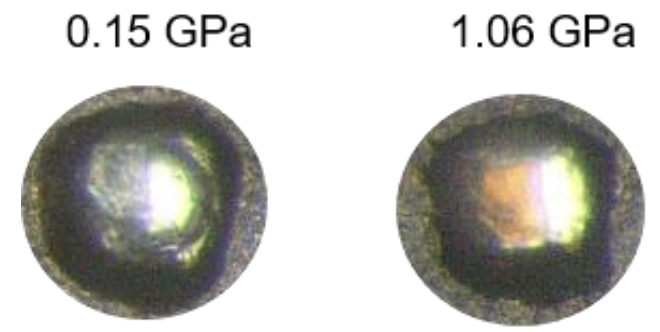

Figure S8: Visual inspection of a single crystal of $2^{\mathrm{Br}} \cdot \mathbf{2} \mathrm{H}_{2} \mathrm{O}$ at 0.15 and $1.06 \mathrm{GPa}$ showing a colour change from yellow to orange indicative of SCO.

Table S5 Single crystal data for $\mathbf{1}^{\mathrm{Cl}} \cdot \mathbf{2} \mathrm{H}_{2} \mathrm{O}$ under hydrostatic pressure.

\begin{tabular}{|c|c|c|c|c|c|}
\hline Parameter & 0 GPa & 0.15 GPa & 0.44 GPa & 0.72 GPa & 0.87 GPa \\
\hline Crystal system & Triclinic & Triclinic & Triclinic & Triclinic & Triclinic \\
\hline Space group & $P-1$ & $P-1$ & $P-1$ & $P-1$ & $P-1$ \\
\hline$a / \AA$ & $7.35210(3)$ & $7.32445(2)$ & $7.3048(6)$ & $7.2466(8)$ & $6.98794(3)$ \\
\hline$b / \AA$ & $7.53600(2)$ & $7.55227(4)$ & $7.5478(10)$ & $7.5389(14)$ & 7.33044(4) \\
\hline$c / \AA$ & $12.40350(3)$ & $12.26713(7)$ & $12.205(7)$ & $12.010(7)$ & $12.11846(9)$ \\
\hline$\alpha / /^{\circ}$ & $106.377(5)$ & $105.977(9)$ & $105.75(3)$ & $105.32(3)$ & 106.217(11) \\
\hline$\beta 1^{\circ}$ & $97.967(5)$ & $97.768(7)$ & $97.433(19)$ & $96.84(2)$ & $98.111(10)$ \\
\hline$\gamma /{ }^{\circ}$ & $90.088(4)$ & $90.136(6)$ & $90.146(9)$ & $90.175(12)$ & $90.211(7)$ \\
\hline Volume/Å ${ }^{3}$ & $652.378(19)$ & $645.82(3)$ & $641.7(4)$ & $627.9(4)$ & $589.50(4)$ \\
\hline $\mathrm{Z}$ & 1 & 1 & 1 & 1 & 1 \\
\hline$\rho_{\text {calc }} \mathrm{g} / \mathrm{cm}^{3}$ & 1.842 & 1.874 & 1.882 & 1.924 & 2.050 \\
\hline$\mu / \mathrm{mm}^{-1}$ & 1.653 & 1.670 & 1.680 & 1.717 & 1.829 \\
\hline Data/restraints/parameters & $2457 / 0 / 175$ & $615 / 68 / 75$ & $614 / 68 / 75$ & $476 / 68 / 75$ & $428 / 68 / 75$ \\
\hline Goodness-of-fit on $\mathrm{F}^{2}$ & 0.9281 & 1.0080 & 0.9933 & 1.0174 & 1.0493 \\
\hline $\begin{array}{l}\text { Final } \mathrm{R} \text { indexes }[\mathrm{I}>=2 \sigma \\
\text { (I)] }\end{array}$ & $0.0581,0.1486$ & $0.1029,0.2425$ & $0.1244,0.2869$ & $0.1091,0.2144$ & $0.1293,0.2898$ \\
\hline Final $\mathrm{R}$ indexes [all data] & $0.0952,0.1667$ & $0.1371,0.2756$ & $0.1719,0.3275$ & $0.1301,0.2339$ & $0.1557,0.3117$ \\
\hline
\end{tabular}




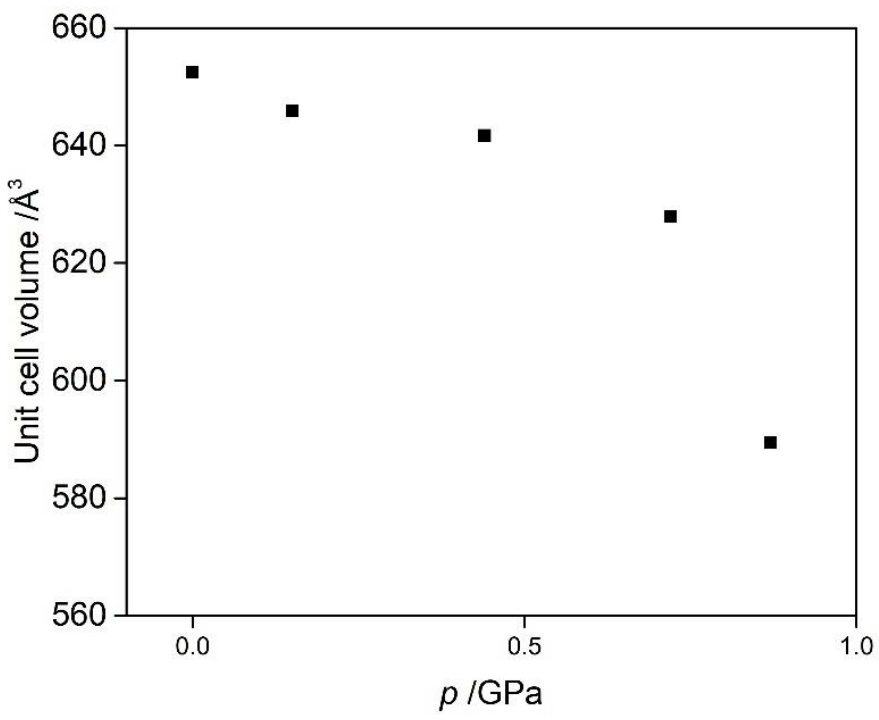

Figure S9. Plot of pressure versus unit cell volume for $\mathbf{1}^{\mathrm{Cl}} \cdot \mathbf{2} \mathrm{H}_{2} \mathrm{O}$ from single crystal X-ray diffraction.

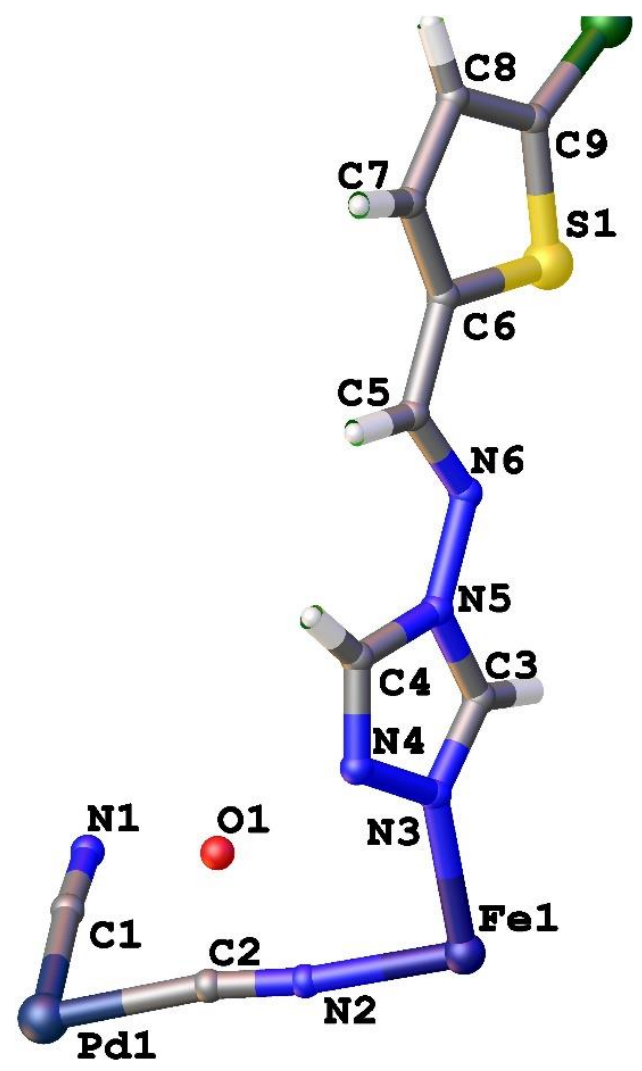

Figure S10. ASU of $\mathbf{1}^{\mathrm{Cl}} \cdot \mathbf{2} \mathrm{H}_{2} \mathrm{O}$ from single crystal X-ray diffraction at $0.87 \mathrm{GPa}$, showing the presence of the water molecule and average Fe-N bond lengths of $c a .1 .96 \AA$. 


\section{References}

1. Halcrow, M. A. Structure: function relationships in molecular spin-crossover complexesChem. Soc. Rev. 2011, 40, 4119-4142; (b) Halcrow, M. A.; Spin-crossover materials: properties and applications, John Wiley \& Sons, 2013 